\title{
On the Consistency of Deferred Acceptance when Priorities are Acceptant Substitutable
}

\author{
Flip Klijn*
}

March 2011

\begin{abstract}
In the context of resource allocation on the basis of responsive priorities, Ergin (2002) identifies a necessary and sufficient condition for the deferred acceptance rule to satisfy a consistency principle. In this note we extend this result to the domain of substitutable priorities, complementing results of Kojima and Manea (2010) and Kumano (2009).
\end{abstract}

JEL classification: D63, C78.

Keywords: deferred acceptance, consistency

\section{Introduction}

In the context of resource allocation on the basis of responsive priorities, Ergin (2002) studies the deferred acceptance rule (Gale and Shapley, 1962). He establishes that Pareto efficiency, groupstrategyproofness, and consistency are equivalent properties. Moreover, he identifies a necessary and sufficient condition on the priority structure to guarantee that the deferred acceptance rule satisfies any of these properties.

Kojima and Manea (2010) point out that in practice priorities may often be non-responsive but substitutable. For the more general framework with substitutable priorities, Kojima and Manea (2010) provide axiomatic characterizations of the deferred acceptance rule. In particular, they extend part of Ergin's (2002) main result by showing that Pareto efficiency, groupstrategyproofness, and Maskin monotonicy are equivalent properties. Kumano (2009) complements these findings of Kojima and Manea (2010) by identifying a necessary and sufficient condition on the priority structure to guarantee that the deferred acceptance rule is Pareto efficient when priorities are substitutable. The combined results of Kumano (2009) and Kojima and Manea (2010) do not completely generalize Ergin's (2002) main result since consistency of the deferred acceptance rule is not dealt with. In this note we fill this gap.

\section{Model}

The notation and description of the deferred acceptance rule is due to Kojima and Manea (2010). There is a set of agents $N$ and a set of proper object types $O$. There is also a null object type, denoted $\emptyset$. Each object $a \in O \cup\{\emptyset\}$ has quota $q_{a} ; q_{\emptyset}=|N|$. Each agent $i$ is allocated exactly one object in $O \cup\{\emptyset\}$. An allocation is a vector $\mu=\left(\mu_{i}\right)_{i \in N}$ assigning object $\mu_{i} \in O \cup\{\emptyset\}$ to agent $i$. For each $a \in O \cup\{\emptyset\}$, we have that $\mu_{a} \equiv\left\{i \in N: \mu_{i}=a\right\}$ satisfies $\left|\mu_{a}\right| \leq q_{a}$.

\footnotetext{
${ }^{*}$ Institute for Economic Analysis (CSIC), Campus UAB, 08193 Bellaterra (Barcelona), Spain; e-mail: flip.klijn@iae.csic.es. Financial support from Plan Nacional I+D+i (ECO2008-04784), the ConsoliderIngenio 2010 (CSD2006-00016) program, the Barcelona Graduate School of Economics and the Government of Catalonia (SGR2009-01142) is gratefully acknowledged.
} 
The preferences of each agent $i$ over the set of objects is represented by a linear order $R_{i}$. We denote by $P_{i}$ the asymmetric part of $R_{i}$, i.e., $a P_{i} b$ if only if $a R_{i} b$ and $a \neq b$. An object $a$ is acceptable to agent $i$ if $a P_{i} \emptyset$. Let $R=\left(R_{i}\right)_{i \in N}$ be the preference profile of all agents. For any $N^{\prime} \subset N$, we write $R_{N^{\prime}}=\left(R_{i}\right)_{i \in N^{\prime}}$.

We denote by $\mathcal{A}$ and $\mathcal{R}$ the sets of allocations and preference profiles, respectively. An allocation rule $\varphi: \mathcal{R} \rightarrow \mathcal{A}$ associates an allocation with each preference profile. At $R$, agent $i$ is assigned object $\varphi_{i}(R)$, and object $a$ is assigned to the set of agents $\varphi_{a}(R)$.

An allocation $\mu$ Pareto dominates another allocation $\mu^{\prime}$ at $R$ if $\mu_{i} R_{i} \mu_{i}^{\prime}$ for all $i \in N$ and $\mu_{i} P_{i} \mu_{i}^{\prime}$ for some $i \in N$. An allocation is Pareto efficient at $R$ if no allocation Pareto dominates it at $R$. An allocation rule $\varphi$ is Pareto efficient if $\varphi(R)$ is Pareto efficient at $R$ for all $R \in \mathcal{R}$. An allocation rule $\varphi$ is group strategy-proof if there exist no $N^{\prime} \subseteq N$ and $R, R^{\prime} \in \mathcal{R}$ such that $\varphi_{i}\left(R_{N^{\prime}}^{\prime}, R_{N \backslash N^{\prime}}\right) R_{i} \varphi_{i}(R)$ for all $i \in N^{\prime}$ and $\varphi_{j}\left(R_{N^{\prime}}^{\prime}, R_{N \backslash N^{\prime}}\right) P_{j} \varphi_{j}(R)$ for some $j \in N^{\prime}$.

A priority for a proper object $a \in O$ is a correspondence $C h_{a}: 2^{N} \rightarrow 2^{N}$ with $C h_{a}\left(N^{\prime}\right) \subseteq N^{\prime}$ and $\left|C h_{a}\left(N^{\prime}\right)\right| \leq q_{a}$ for all $N^{\prime} \subseteq N$. We make the following two assumptions on each priority. First, $C h_{a}$ is substitutable, i.e., for all $N^{\prime} \subset N^{\prime \prime} \subseteq N, C h_{a}\left(N^{\prime \prime}\right) \cap N^{\prime} \subseteq C h_{a}\left(N^{\prime}\right)$. Second, $C h_{a}$ is acceptant, i.e., for all $N^{\prime} \subseteq N,\left|C h_{a}\left(N^{\prime}\right)\right|=\min \left(q_{a},\left|N^{\prime}\right|\right)$. Let $C h \equiv\left(C h_{a}\right)_{a \in O}$ denote the (acceptant substitutable) priority structure.

An allocation $\mu$ is individually rational at $R$ if for all $i \in N, \mu_{i} R_{i} \emptyset$. The allocation $\mu$ is blocked by a pair $(i, a) \in N \times O$ at $(R, C h)$ if $a P_{i} \mu_{i}$ and $i \in C h_{a}\left(\mu_{a} \cup\{i\}\right)$. An allocation $\mu$ is stable at $(R, C h)$ if it is individually rational at $R$ and is not blocked by any pair $(i, a) \in N \times O$ at $(R, C h)$. Since $C h$ is substitutable, the so-called deferred acceptance rule, denoted $\varphi^{C h}$, yields a stable allocation $\varphi^{C h}(R)$ at $(R, C h)$ (Roth and Sotomayor, 1990, proof of Theorem 6.5). The allocation $\varphi^{C h}(R)$ is obtained through the deferred acceptance algorithm as follows.

STEP 1: Each agent applies to his most preferred acceptable object under $R$ (if any). Let $\tilde{N}_{a}^{1}$ be the set of agents applying to object $a$. Object $a$ tentatively accepts the agents in $N_{a}^{1} \equiv C h_{a}\left(\tilde{N}_{a}^{1}\right)$, and rejects the agents in $\tilde{N}_{a}^{1} \backslash N_{a}^{1}$.

STEP $t, t \geq 2$ : Each agent who was rejected at step $t-1$ applies to his next preferred acceptable object under $R$ (if any). Let $\tilde{N}_{a}^{t}$ be the new set of agents applying to object $a$. Object $a$ tentatively accepts the agents in $N_{a}^{t} \equiv C h_{a}\left(N_{a}^{t-1} \cup \tilde{N}_{a}^{t}\right)$, and rejects the agents in $\left(N_{a}^{t-1} \cup\right.$ $\left.\tilde{N}_{a}^{t}\right) \backslash N_{a}^{t}$

The algorithm terminates when each agent is either tentatively accepted by some object or has been rejected by every object that is acceptable to him. Each agent tentatively accepted by a proper object at the last step is assigned that object, and all other agents are assigned the null object. The allocation reached by the deferred acceptance rule is the agent-optimal stable allocation at $(R, C h)$. That is, $\varphi^{C h}(R)$ is stable at $(R, C h)$, and all agents weakly prefer $\varphi^{C h}(R)$ to any other stable allocation at $(R, C h)$ (Roth and Sotomayor, 1990, Theorem 6.8).

Since efficiency is one of the goals of the social planner in resource allocation problems, Kojima and Manea (2010) develop necessary and sufficient conditions for the efficiency of the deferred acceptance rules.

Proposition 1. (Kojima and Manea, 2010, Proposition 1)

The following properties are equivalent.

(i) $\varphi^{C h}$ is Pareto efficient.

(ii) $\varphi^{C h}$ satisfies Maskin monotonicity. ${ }^{1}$

(iii) $\varphi^{C h}$ is group strategy-proof.

Kumano (2009) derives a generalization of the acyclicity condition introduced by Ergin (2002)

\footnotetext{
${ }^{1}$ For the definition of Maskin monotonicity we refer to Kojima and Manea (2010) and Maskin (1999).
} 
and shows that it is a sufficient and necessary condition on the priority structure to guarantee that $\varphi^{C h}$ satisfies either of the properties in Proposition 1.

A cycle is constituted of distinct $i, j, k \in N$ and $a, b \in O$ such that that there are disjoint (and possibly empty) sets $N_{a}, N_{b} \subseteq N \backslash\{i, j, k\}$ satisfying

(C) $j \notin C h_{a}\left(N_{a} \cup\{i, j\}\right), k \notin C h_{a}\left(N_{a} \cup\{j, k\}\right)$, and $i \notin C h_{b}\left(N_{b} \cup\{k, i\}\right)$.

(S) $\left|N_{a}\right|=q_{a}-1$ and $\left|N_{b}\right|=q_{b}-1$.

If there is no cycle, then we say that the priority structure is acyclical.

Proposition 2. (Kumano, 2009, Proposition 1)

The following properties are equivalent.

(i) $\varphi^{C h}$ is Pareto efficient.

(iv) $C h$ is acyclical.

Combining Propositions 1 and 2 yields a partial generalization of the main result of Ergin (2002, Theorem 1), who considers acceptant responsive priority structures (which are a strict subclass of acceptant substitutable priority structures). ${ }^{2}$ The generalization of Ergin (2002, Theorem 1) is not complete since Kojima and Manea (2010) and Kumano (2009) do not consider the consistency of the (extended) deferred acceptance rule. In the next section we fill this gap.

\section{Result}

We first introduce consistency. Let $C h$ be a priority structure. An economy is a triple $\left(N^{\prime}, q^{\prime}, R_{N^{\prime}}\right)$ where $N^{\prime} \subseteq N$ and $q^{\prime} \equiv\left(q_{a}^{\prime}\right)_{a \in O}$ with $q_{a}^{\prime} \leq q_{a}$ for all $a \in O$. The extended deferred acceptance rule, denoted $\tilde{\varphi}^{C h}$, associates with each economy $\left(N^{\prime}, q^{\prime}, R_{N^{\prime}}\right)$ the allocation that is obtained by applying the (accordingly adapted) deferred acceptance algorithm to $\left(N^{\prime}, q^{\prime}, R_{N^{\prime}}\right)$ and the restricted priority structure $\left(C h_{a \mid 2^{N^{\prime}}}\right)_{a \in O}$. Given the original economy $(N, q, R)$, an allocation $\mu$ for $(N, q, R)$, and a subset of agents $N^{\prime} \subset N$, the reduced economy of $(N, q, R)$ with respect to $\mu$ and $N^{\prime}$ is the economy $\left(N^{\prime}, q^{\mu}, R_{N^{\prime}}\right)$ where for all $a \in O, q_{a}^{\mu} \equiv q_{a}-\left|\mu_{a} \backslash N^{\prime}\right|$. Consistency of the extended deferred acceptance rule requires that once an allocation for the original economy is determined and some agents receive their assigned objects before the others, the rule should not change the assigned objects of the remaining agents in the reduced economy. Formally, a priority structure $C h$ induces a consistent extended deferred acceptance rule $\tilde{\varphi}^{C h}$ if for each $N^{\prime} \subset N$ and each preference profile $R, \mu_{\mid N^{\prime}}=\tilde{\varphi}^{C h}\left(N^{\prime}, q^{\mu}, R_{N^{\prime}}\right)$ where $\mu=\tilde{\varphi}^{C h}(N, q, R)$. The following result completes the generalization of Ergin's (2002) main result.

Proposition 3. Each property in Propositions 1 and 2 is equivalent to the consistency of the extended deferred acceptance rule $\tilde{\varphi}^{C h}$.

Proof. In view of (i) $\Leftrightarrow$ (iii) in Proposition 1, it is sufficient to prove that (i) $\Rightarrow \tilde{\varphi}^{C h}$ is consistent $\Rightarrow$ (iii). The proof is an adaptation of Ergin's (2002) proof.

(i) $\Rightarrow \tilde{\varphi}^{C h}$ is consistent: Assume $\tilde{\varphi}^{C h}$ is not consistent. Then there are $N^{\prime} \subset N$ and $R$ such that $\mu_{\mid N^{\prime}} \neq \tilde{\varphi}^{C h}\left(N^{\prime}, q^{\mu}, R_{N^{\prime}}\right)$ where $\mu=\tilde{\varphi}^{C h}(N, q, R)$. Since $\mu=\tilde{\varphi}^{C h}(N, q, R)=\varphi(R)$ is a stable allocation in the original economy $(N, q, R)$, it immediately follows that $\mu_{\mid N^{\prime}}$ is stable in the reduced economy $\left(N^{\prime}, q^{\mu}, R_{N^{\prime}}\right)$. Since priorities are substitutable it follows from Roth and Sotomayor (1990, Theorem 6.8) that $\tilde{\varphi}^{C h}\left(N^{\prime}, q^{\mu}, R_{N^{\prime}}\right)$ Pareto dominates $\mu_{\mid N^{\prime}}$ in the reduced economy $\left(N^{\prime}, q^{\mu}, R_{N^{\prime}}\right)$. Therefore, in the original economy, the allocation $\nu$ defined by

$$
\nu_{i} \equiv \begin{cases}\tilde{\varphi}_{i}^{C h}\left(N^{\prime}, q^{\mu}, R_{N^{\prime}}\right) & \text { if } i \in N^{\prime} \\ \mu_{i} & \text { if } i \in N \backslash N^{\prime}\end{cases}
$$

\footnotetext{
${ }^{2}$ A priority structure $C h$ is responsive if for each $a \in O$, there is a linear order $R_{a}$ on $N$ such that for each $N^{\prime} \subseteq N, C h_{a}\left(N^{\prime}\right)$ equals the set of $\min \left(q_{a},\left|N^{\prime}\right|\right)$ top ranked agents in $N^{\prime}$ under $R_{a}$.
} 
Pareto dominates $\mu$. Hence, $\varphi^{C h}$ is not Pareto efficient.

$\tilde{\varphi}^{C h}$ is consistent $\Rightarrow$ (iii): Assume $\tilde{\varphi}^{C h}$ is consistent. From the proof of Lemma 1 in Pápai (2000) it follows that strategy-proofness and nonbossiness imply group strategyproofness. ${ }^{3}$ Since priorities are acceptant substitutable, it follows that for any object $a \in O$ and any two sets of agents $N^{\prime} \subset N^{\prime \prime} \subseteq N,\left|C h_{a}\left(N^{\prime}\right)\right|=\min \left(q_{a},\left|N^{\prime}\right|\right) \leq \min \left(q_{a},\left|N^{\prime \prime}\right|\right)=\left|C h_{a}\left(N^{\prime \prime}\right)\right|$. Therefore, the priorities satisfy the law of demand (Hatfield and Milgrom, 2005, page 923). Hence, by Theorems 11 and 12 in Hatfield and Milgrom (2005), $\varphi^{C h}$ is strategy-proof. To complete the proof we show that $\varphi^{C h}$ is nonbossy. Let $i \in N$ and $R, R^{\prime} \in \mathcal{R}$ be such that $\varphi_{i}^{C h}(R)=\varphi_{i}^{C h}\left(R_{i}^{\prime}, R_{N \backslash\{i\}}\right)$. Let $\mu \equiv \varphi^{C h}(R)$ and $\nu \equiv \varphi^{C h}\left(R_{i}^{\prime}, R_{N \backslash\{i\}}\right)$. Note that since $\mu_{i}=\nu_{i}$, the reduced economies $\left(N \backslash\{i\}, q^{\mu}, R_{N \backslash\{i\}}\right)$ and $\left(N \backslash\{i\}, q^{\nu}, R_{N \backslash\{i\}}\right)$ are the same. Hence, $\tilde{\varphi}^{C h}\left(N \backslash\{i\}, q^{\mu}, R_{N \backslash\{i\}}\right)=\tilde{\varphi}^{C h}\left(N \backslash\{i\}, q^{\nu}, R_{N \backslash\{i\}}\right)$. On the other hand, by consistency, $\mu_{\mid N \backslash\{i\}}=\tilde{\varphi}^{C h}\left(N \backslash\{i\}, q^{\mu}, R_{N \backslash\{i\}}\right)$ and $\nu_{\mid N \backslash\{i\}}=\tilde{\varphi}^{C h}\left(N \backslash\{i\}, q^{\nu}, R_{N \backslash\{i\}}\right)$. Hence, $\mu_{\mid N \backslash\{i\}}=\nu_{\mid N \backslash\{i\}}$, which together with $\mu_{i}=\nu_{i}$ implies that $\varphi^{C h}(R)=\mu=\nu=\varphi^{C h}\left(R_{i}^{\prime}, R_{N \backslash\{i\}}\right)$. Hence, $\varphi^{C h}$ is nonbossy.

\section{References}

Ergin, H. İ. (2002): "Efficient Resource Allocation on the Basis of Priorities," Econometrica, 70(6), 2489-2497.

Gale, D., and L.S. Shapley (1962): "College Admissions and the Stability of Marriage," The American Mathematical Monthly, 69(1), 9-15.

Hatfield, J.W., and P. Milgrom (2005): "Matching with Contracts," American Economic Review, 95(4), 913-935.

Kojima, F., and M. Manea (2010): “Axioms for Deferred Acceptance," Econometrica, 78(2), 633-653.

Kumano, T. (2009): "Efficient Resource Allocation under Acceptant Substitutable Priorities," Working paper, Washington University in St. Louis.

Maskin, E. (1999): "Nash Equilibrium and Welfare Optimality," Review of Economic Studies, 66(1), 23-38.

Pápai, S. (2000): "Strategyproof Assignment by Hierarchical Exchange," Econometrica, 68(6), 1403-1433.

Roth, A.E., and M.A.O. Sotomayor (1990): Two-Sided Matching: A Study in Game-Theoretic Modeling and Analysis. Econometric Society Monograph Series. New York: Cambridge University Press.

\footnotetext{
${ }^{3}$ An allocation rule $\varphi$ is strategy-proof if there exist no $i \in N$ and $R, R^{\prime} \in \mathcal{R}$ such that $\varphi_{i}\left(R_{i}^{\prime}, R_{N \backslash\{i\}}\right) P_{i} \varphi_{i}(R)$. An allocation rule $\varphi$ is nonbossy if for all $i \in N$ and all $R, R^{\prime} \in \mathcal{R}, \varphi_{i}(R)=\varphi_{i}\left(R_{i}^{\prime}, R_{N \backslash\{i\}}\right)$ implies $\varphi\left(R_{i}^{\prime}, R_{N \backslash\{i\}}\right)=$ $\varphi(R)$.
} 